Supporting Information

\title{
Redox-Driven Spontaneous Double Emulsion
}

Ruiting Li, Zhen Wang, Xinglei Tao, Jichen Jia, Xiaodong Lian, and Yapei Wang* Department of Chemistry, Renmin University of China, Beijing 100872, China 


\section{EXPERIMENTAL SECTION}

\section{Materials:}

Polystyrene-b-polybutadiene-b-polystyrene (SBS, D1101J) and poly(styrene-ethylene/butylenestyrene) (SEBS) were obtained from Kraton. Polyvinyl alcohol (PVA) was provided by Lyntech. Iodine $\left(\mathrm{I}_{2}\right)$ was purchased from Shanghai Aladdin Bio-Chem Technology Co., Ltd. Chlorobenzene was purchased from Sigma-Aldrich. Water was obtained from a Milli-Q Integral Water Purification System $\left(18.2 \mathrm{M} \Omega \mathrm{cm}^{-1}\right)$.

\section{Characterization methods:}

An inverted microscope (Leica DMi8) was used to record bright-field images of the emulsions in aqueous solution. Fourier transform infrared (FTIR) spectra were obtained on a Bruker IFS66v FTIR spectrometer. Electrochemical workstation (CHI-660e Shanghai Chenhua Co.) was used to monitor the conductivity changes of SBS and iodine dissolved in chlorobenzene with different temperature. ${ }^{1} \mathrm{H}$ NMR spectra were obtained by a Bruker $400 \mathrm{MHz}$ spectrometer. Water contact angles were measured on an optical contact angle measuring device (KRÜSS Drop Shape Analyzer DSA30). X-ray photoelectron spectroscopy was performed by Thermo Scientific ESCALab250 to identify the exact species of iodine and carbon at different doping stages.

\section{Fabrication of spontaneous emulsion:}

Water-in-oil emulsions were produced by dynamic interfacial printing technique (DIP-3C-01, Hongguang Tech.). The aqueous phase is composed of water containing $0.5 \mathrm{wt} \%$ polyvinyl alcohol (PVA), and the oil phase contains $2 \mathrm{wt} \%$ SBS dissolved in chlorobenzene with addition of iodine with different concentration.

\section{Mathematical clarification of mechanism of forming the spontaneous double emulsions:}

Quantitively, as is known, the Laplace pressure caused by the interfacial tension between the interior of a droplet and the external oil phase can be described by the following equation:

$$
\Delta \mathrm{P}=2 \gamma / \mathrm{r}
$$

where $\Delta \mathrm{P}$ is the Laplace pressure, $\gamma$ is the interfacial tension, and $\mathrm{r}$ is the radius of the droplet. When the aqueous phase within the oil droplets of double emulsions is treated as an ideal dilute solution with a concentration $c_{i}$ of monovalent salt which is SBS-iodide complex in our case, the osmotic pressure $\prod_{i}$ resulting from the high ion concentration within the aqueous droplets can be approximated according to the following equation: 


$$
\Pi_{\mathrm{i}}=2 \mathrm{c}_{\mathrm{i}} \mathrm{RT}
$$

where $\mathrm{R}$ is the gas constant which is $8.314 \mathrm{kPa} \cdot \mathrm{L} \cdot \mathrm{K}^{-1} \cdot \mathrm{mol}^{-1}$ and $\mathrm{T}$ is the absolute temperature. Similarly, the osmotic pressure $\Pi_{\mathrm{o}}$ in the bulk aqueous phase can be derived as

$$
\Pi_{\mathrm{o}}=2 \mathrm{c}_{\mathrm{o}} \mathrm{RT}
$$

where $c_{o}$ is the concentration of salt in the external aqueous solution. Obviously, the osmotic pressure difference $\Pi$ between the internal water droplets and the external water phase can be calculated as

$$
\Pi=\Pi_{i}-\Pi_{o}=2 c_{i} R T-2 c_{0} R T=2\left(c_{i}-c_{o}\right) R T
$$

At the very beginning, there is no any ionic compounds existing in the external bulk aqueous solution because only the non-ionic surfactant PVA was used as the emulsifiers. So, $\Pi_{\mathrm{o}}$ is rationally determined as 0 , and the original very small internal water droplets can only be formed under the condition of $\Delta \mathrm{P}<\Pi=\Pi_{\mathrm{i}}$. In this regard, we can know:

$$
2 \gamma_{0} / r_{0}<2 c_{i 0} R T
$$

which can be also written as:

$$
\gamma_{0} / \mathrm{r}_{0}<\mathrm{c}_{\mathrm{i} 0 \mathrm{RT}}
$$

where $\gamma_{0}$ is the interfacial tension between water droplets and oil phase, $r_{0}$ is the radius of the internal water droplets, and $\mathrm{c}_{\mathrm{i} 0}$ is the concentration of salt in the internal water droplets all when the internal water droplets were initially formed.

We define the contrast between the osmotic pressure difference and the Laplace pressure difference as the driving force $\mathrm{F}$ for the formation of spontaneous double emulsions, according to the previous deduction, we can know:

$$
\mathrm{F}=\Pi-\Delta \mathrm{P}=2\left(\mathrm{c}_{\mathrm{i}}-\mathrm{c}_{\mathrm{o}}\right) \mathrm{RT}-2 \gamma / \mathrm{r}=2\left[\left(\mathrm{c}_{\mathrm{i}}-\mathrm{c}_{\mathrm{o}}\right) \mathrm{RT}-\gamma / \mathrm{r}\right]
$$

where the value of $\mathrm{F}$ can be affected by four main parameters which are the concentration of salt $\mathrm{c}_{\mathrm{i}}$ in the internal water droplets, $c_{o}$ in the external water phase, the interfacial tension $\gamma$ between water droplets and oil phase, and the radius $r$ of the internal water droplets.

As is known, the interfacial tension $\gamma$ between water droplets and oil phase is closely related to the type and concentration of the interfacial stabilizer. In our system, given the type of emulsifier as SBS-iodide complex, as the doping process between SBS and iodine goes, the SBS-iodide complexes are being produced and the concentration of this emulsifier increases constantly. It is assumed that the timely interfacial tension $\gamma$ would decrease all the time, always less than the 
original interfacial tension $\gamma_{0}$. To confirm this assumption, the interfacial tension $\gamma$ was measured and tracked by an optical contact angle measuring device (KRÜSS drop shape analyzer DSA30). As shown in Figure S1, SBS-I ${ }_{2}$ complexes could remarkably lower the interfacial tension because as discussed in the manuscript they possess good amphiphilicity and can be well-assembled at the oil/water interface to maintain the stabilization of this interface. And in our observation window, the interfacial tension $\gamma$ did decrease with the increase of reaction time all the time because more emulsifiers were produced for better stabilization of water/oil interface. Combined with the increasing radius of the internal water droplets as observed by us and shown in the manuscript, it can be obtained that:

$$
\mathrm{F}=2\left[\left(\mathrm{c}_{\mathrm{i}}-\mathrm{c}_{\mathrm{o}}\right) \mathrm{RT}-\gamma / \mathrm{r}\right]>2\left[\left(\mathrm{c}_{\mathrm{i}}-\mathrm{c}_{\mathrm{o}}\right) \mathrm{RT}-\gamma_{0} / \mathrm{r}_{0}\right]>2\left[\left(\mathrm{c}_{\mathrm{i}}-\mathrm{c}_{\mathrm{o}}\right) \mathrm{RT}-\mathrm{c}_{\mathrm{i} 0} \mathrm{RT}\right]
$$

so,

$$
\mathrm{F}>2\left(\mathrm{c}_{\mathrm{i}}-\mathrm{c}_{\mathrm{o}}-\mathrm{c}_{\mathrm{i} 0}\right) \mathrm{RT}
$$

Providing that the SBS-iodide salt would not diffuse out of the water droplets and into the external bulk water phase, the relationship above can be simplified as

$$
\mathrm{F}>2\left(\mathrm{c}_{\mathrm{i}}-\mathrm{c}_{\mathrm{i} 0}\right) \mathrm{RT}
$$

As we know, the SBS-iodide salt will be produced all the time as the reaction goes. So, at any time, the concentration $c_{i}$ within the internal water droplets would be more than that $c_{i 0}$ at the beginning of this doping reaction between SBS and iodine if the production rate of SBS-iodide complexes is more than the transport rate of water. In this case, the driving force $\mathrm{F}$ would be positive forever, the internal water droplets would expand, and the spontaneous double emulsions would be formed until this reaction is stopped or the reagent SBS or iodine is run out of when the equilibrium will be reached. However, if the production rate of SBS-iodide complexes is not comparable to the transport rate of water, the exact process would vary for every pair of rates.

On the other hand, if the SBS-iodide salt could diffuse out, according to what has been discussed above, the driving force $\mathrm{F}$ can be balanced sometime when

$$
\left(\mathrm{c}_{\mathrm{ix}}-\mathrm{c}_{\mathrm{ox}}\right) \mathrm{RT}=\gamma_{\mathrm{x}} / \mathrm{r}_{\mathrm{x}}
$$

Whether or not this could be reached and achieved depends on the relative rate of the transport of water and salt, as well as the decreasing rate of $\gamma / \mathrm{r}$. More complex measurements and calculations need to be done to clarify these special cases. However, the formation of the spontaneous emulsions is driven by the osmotic pressure difference between the internal water droplets and external water 
phase for sure. When the resulting osmotic pressure difference is comparable to the Laplace pressure difference of the internal aqueous droplets, the equilibrium would be reached and the internal water droplets would stop expanding.

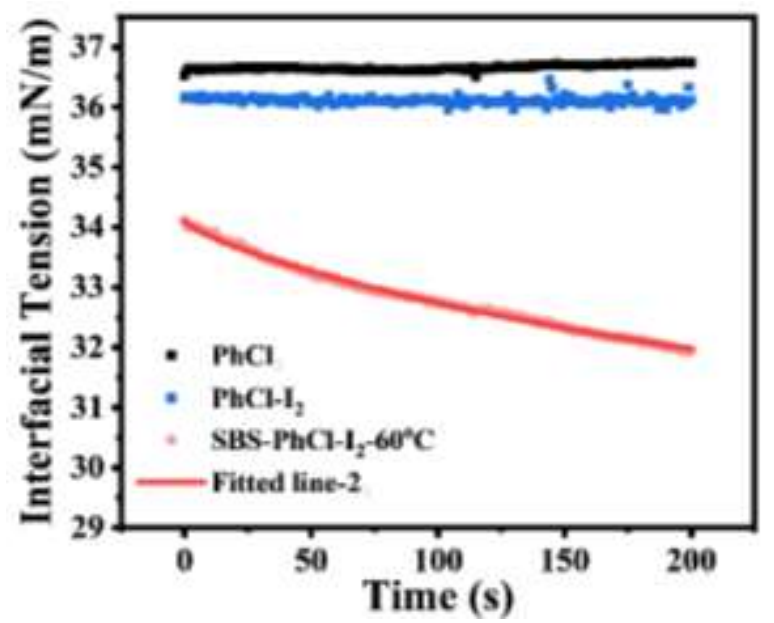

Figure S1. Record of interfacial tension over time between aqueous solution and chlorobenzene solution containing $\mathrm{I}_{2}$ and $\mathrm{SBS}-\mathrm{I}_{2}$. The interfacial tension was fitted to an empirical model to estimate the equilibrium value of the interfacial tension $\gamma$ eqb $=\gamma(t \rightarrow \infty) .{ }^{1,2}$

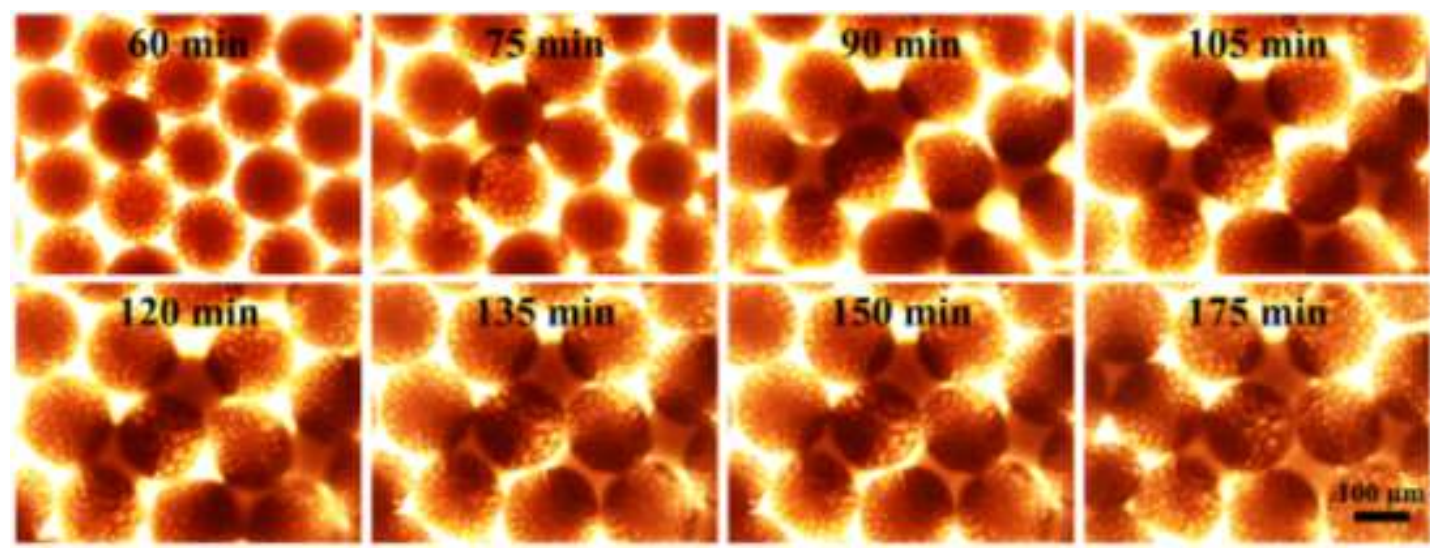

Figure S2. Microscopy images of the emulsions with the oil phase containing 2 wt $\%$ SBS and iodine of $100 \mathrm{mg} / \mathrm{mL}$, and the water phase containing $0.5 \mathrm{wt} \%$ PVA after incubated under $60^{\circ} \mathrm{C}$ for different time. The observation was carried out to investigate the evolution of the spontaneous double emulsions after 60 min until almost 3 hours. As time went and the reaction progressed, more water flew into the oil droplets as a result of the osmotic pressure difference. The shape of double emulsions became irregular gradually. At this stage, there is no point in measuring the size of these emulsions and comparing them in Figure 1e. 

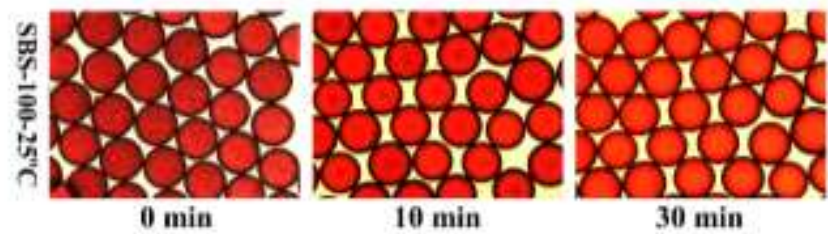

$30 \mathrm{~min}$

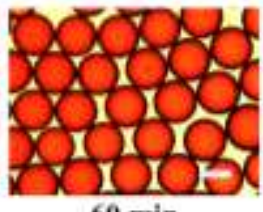

$60 \mathrm{~min}$

Figure S3. Optical microscopy images of emulsions containing PVA aqueous solution and the oil phase containing SBS and iodine of $100 \mathrm{mg} / \mathrm{mL}$ at room temperature under different time.

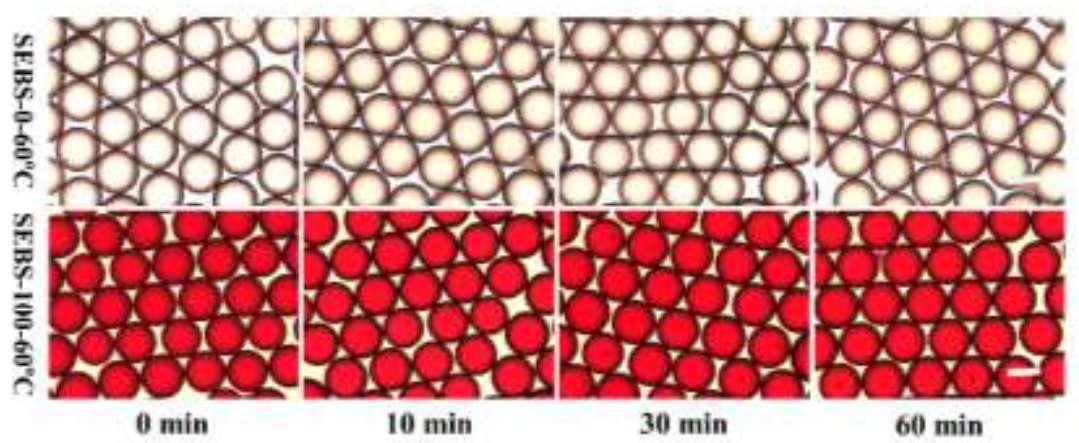

Figure S4. Optical microscopy images of SEBS droplets with different iodine concentrations and different doping time.

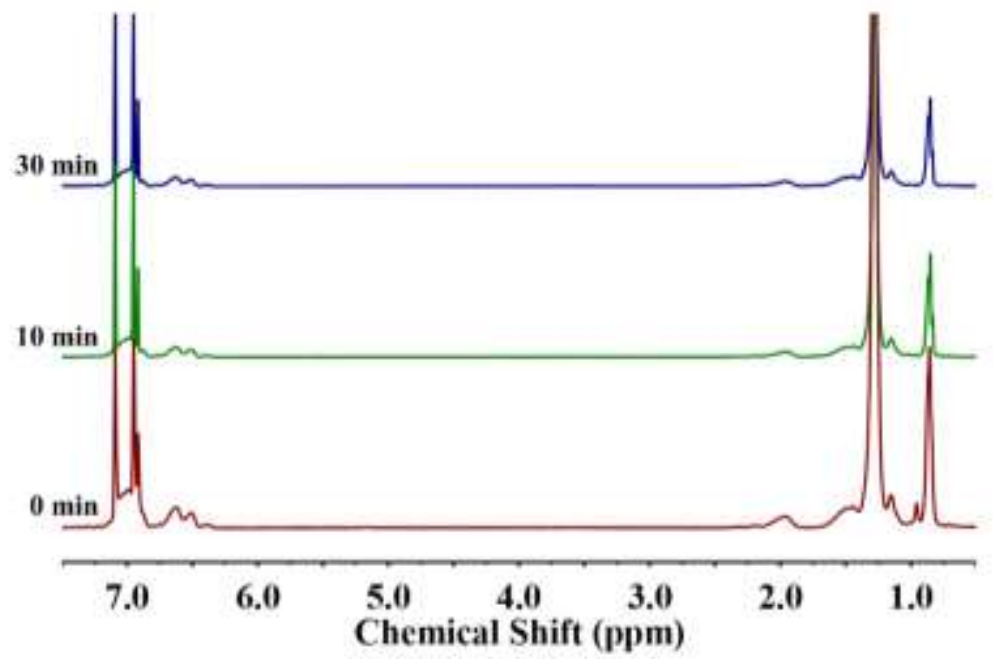

Figure S5. H-NMR spectra of SEBS with iodine doping for different time. 


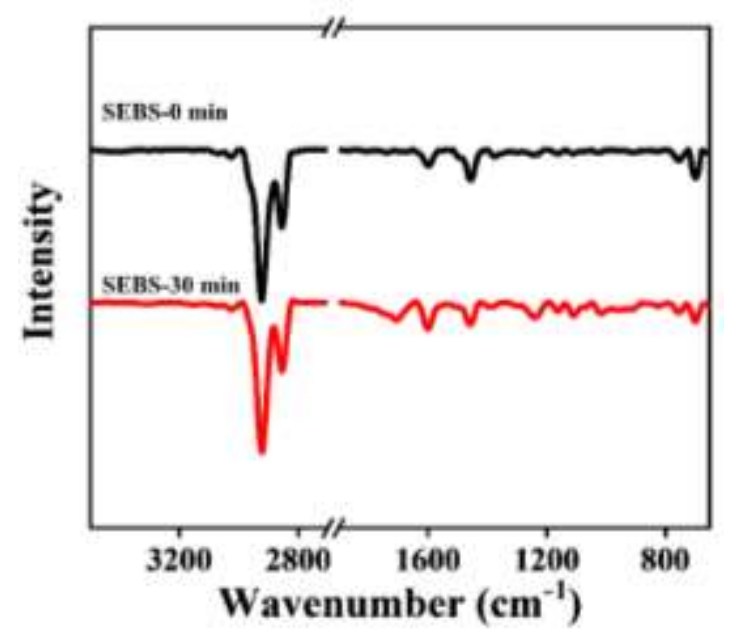

Figure S6. FTIR spectra of SEBS doped with iodine for different time.

Movie S1. Evolution of the emulsion droplets at $60^{\circ} \mathrm{C}$ with the oil phase containing $2 \mathrm{wt} \% \mathrm{SBS}$ and iodine of $100 \mathrm{mg} / \mathrm{mL}$, and the water phase containing $0.5 \mathrm{wt} \%$ PVA.

Movie S2. Evolution of the emulsion droplets at room temperature with the oil phase containing 2 wt $\%$ SBS and iodine of $100 \mathrm{mg} / \mathrm{mL}$, and the water phase containing $0.5 \mathrm{wt} \%$ PVA.

Movie S3. Evolution of the emulsion droplets at $60^{\circ} \mathrm{C}$ with the oil phase containing $2 \mathrm{wt} \% \mathrm{SBS}$ and iodine of $100 \mathrm{mg} / \mathrm{mL}$, and the water phase containing $0.5 \mathrm{wt} \% \mathrm{PVA}$ and $\mathrm{NaCl}$ of $20 \mathrm{mg} / \mathrm{mL}$.

The entire movies (Movie S1-S3) which lasted for 60 min have been speeded up from 60 min to $25 \mathrm{~s}$.

\section{REFERENCES}

1. Hua, X. Y.; Rosen, M. J. Dynamic Surface-Tension of Aqueous Surfactant Solutions .1. Basic Parameters. J. Colloid Interf. Sci. 1988, 124 (2), 652-659.

2. Zarzar, L. D.; Sresht, V.; Sletten, E. M.; Kalow, J. A.; Blankschtein, D.; Swager, T. M. Dynamically Reconfigurable Complex Emulsions Via Tunable Interfacial Tensions. Nature 2015, 518 (7540), 520-524. 